\title{
Proof of Beal's Conjecture
}

\author{
PROF. DR. K. RAJA RAMA GANDHI ${ }^{1}$ AND REUVEN TINT ${ }^{2}$ \\ Resource person in Math for Oxford University Press and Professor at BITS-Vizag ${ }^{1}$ \\ Number Theorist, |srael $\left.\right|^{2}$ \\ Email: editor126@gmail.com, reuven.tint@gmail.com
}

Abstract. We give the complete proof of Beal's conjecture.

\section{Theorem.}

If

$$
A^{x}+B^{y}=C^{z}
$$

where - $A, B, C, x, y, z$ are natural numbers and $x, y, z>2$, then $A, C$ ve a co nmon prime factor.

\section{Proof.}

1. We have the identity:

$$
\left[A\left(A^{x}+B^{x}\right)\right]^{x}+\left[B\left(A^{x}+B^{x}\right)\right]^{x}
$$

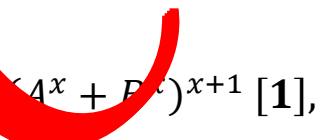

where $A, B, x=0,1,2,3, \ldots$.

1.1. If $\left(A^{x}+B^{x}\right)$ is a prime num 1 thesp ct to [1]. We find then:

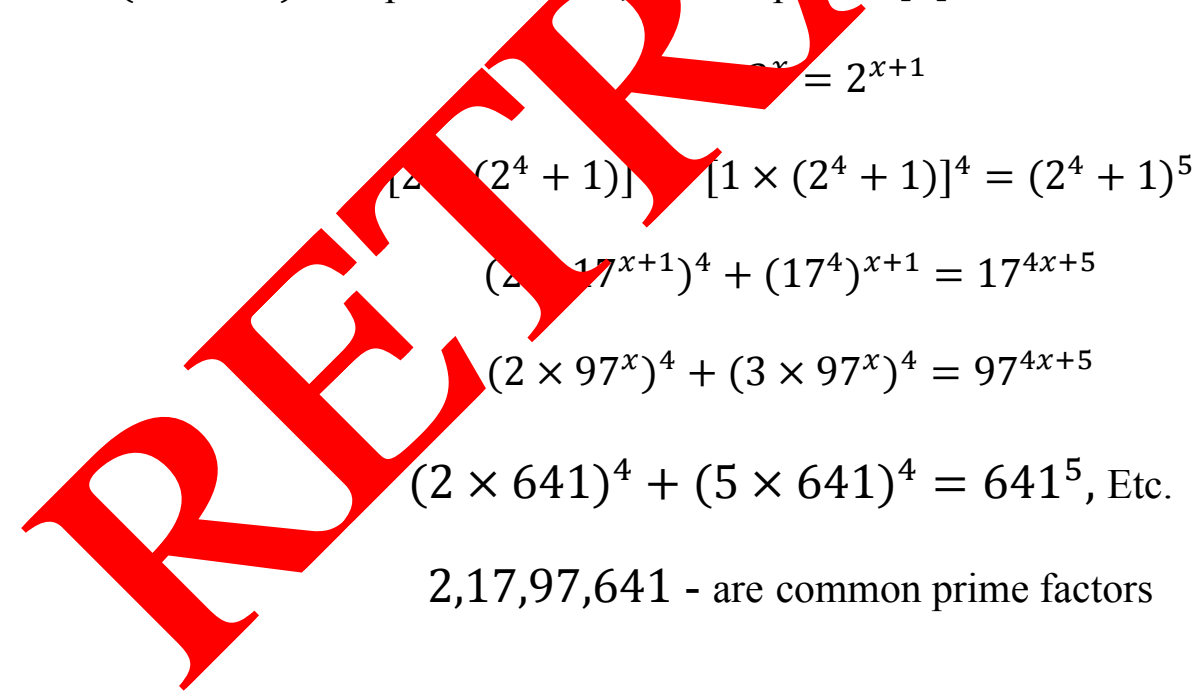

2.

$$
\begin{aligned}
& A^{x}+B^{y}=C^{z} \\
& 1 \times x-0 \times y z=x \\
& 1 \times y-0 \times x z=y \\
& 1 \times z-0 \times x y=z \\
& \left(A^{1} \times B^{0 \times y} \times C^{0 \times z}\right)^{x}+\left(A^{0 \times x} \times B^{1} \times C^{0 \times z}\right)^{y}=\left(A^{0 \times y} \times B^{0 \times x} \times C^{1}\right)^{z}
\end{aligned}
$$


If $C=P-$ is a prime number, then

$$
\begin{gathered}
\left(A \times P^{y}\right)^{x}+\left(B \times P^{x}\right)^{y} \equiv P^{x y+z}[2] \\
2^{2}+1=5,2^{4}+1=17,2^{8}+1=257 \\
\left(2 \times 17^{2 x}\right)^{3}+\left(3 \times 17^{3 x}\right)^{2} \equiv 17^{6 x+1} \\
\left(2 \times 89^{4 x}\right)^{3}+\left(3 \times 89^{3 x}\right)^{4} \equiv 89^{12 x+1} \\
\left(3^{3 y}\right)^{x}+\left(2 \times 3^{x y}\right)^{3} \equiv 3^{3 x y+2}
\end{gathered}
$$

3. If the following condition hold $x=a b$, then we obtain from [1]:

$$
\left[A^{b}\left(A^{a b}+B^{a b}\right)^{b}\right]^{a}+\left[B^{a}\left(A^{a b}+B^{a b}\right)^{a}\right]^{b} \equiv\left(A^{a b}\right.
$$

4. Therefore, the identities [1], [2], [3] with the statement list a 1 , give a comprehensive understanding solvability of equation

in positive integers.

$$
A_{1}^{x}+B_{1}^{y}=C_{1}^{z}
$$

\section{References:}

[1] PROF. DR. K. RAJA RAMA GANDHI, Leuver Tin

"The reproductive solution for Fermat's Last theorem (eleme atary pect)- - rct proof.

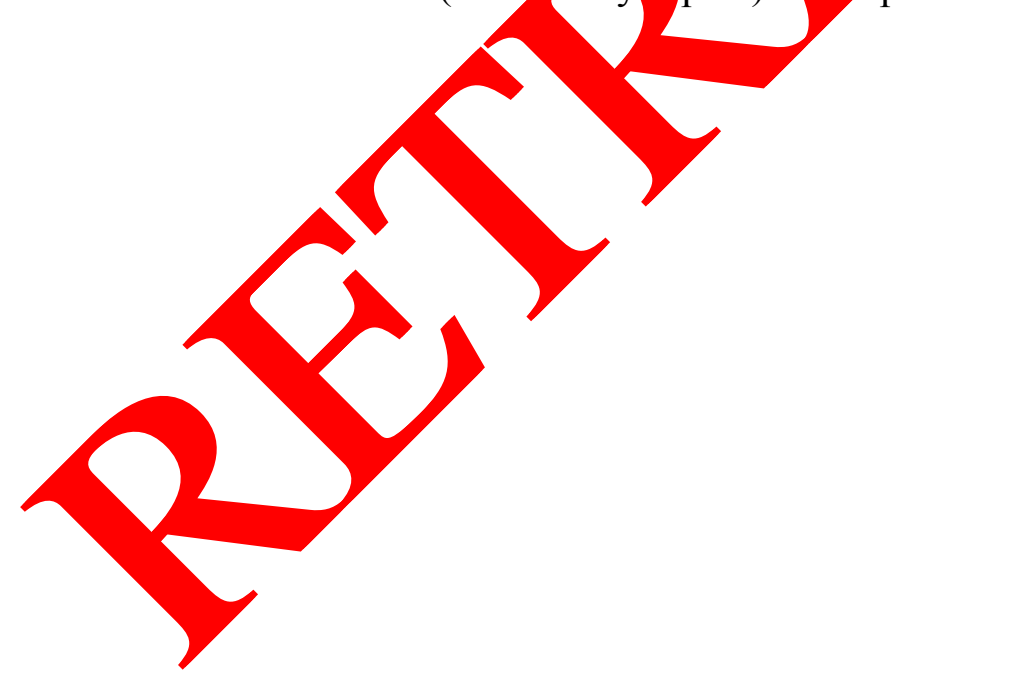

